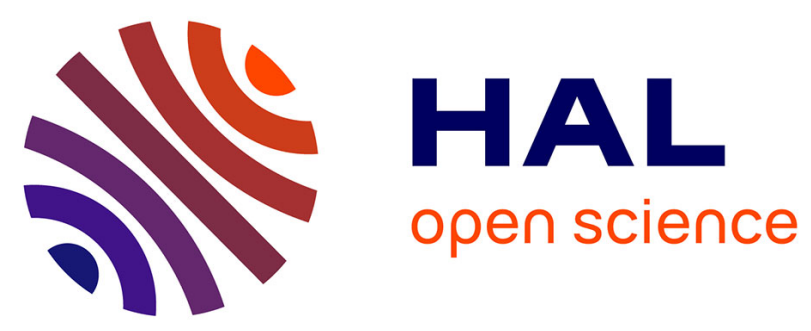

\title{
Dimensioning a product in preliminary design through different exploration techniques
}

Bernard Yannou, Nadège Troussier, Alaa Chateauneuf, Nassim Boudaoud, Dominique Scaravetti

\section{- To cite this version:}

Bernard Yannou, Nadège Troussier, Alaa Chateauneuf, Nassim Boudaoud, Dominique Scaravetti. Dimensioning a product in preliminary design through different exploration techniques. International Journal of Product Development, 2009, 9 (1/2/3), pp.140-163. 10.1504/IJPD.2009.026177 . hal00748725

\section{HAL Id: hal-00748725 \\ https://hal.science/hal-00748725}

Submitted on 17 Mar 2013

HAL is a multi-disciplinary open access archive for the deposit and dissemination of scientific research documents, whether they are published or not. The documents may come from teaching and research institutions in France or abroad, or from public or private research centers.
L'archive ouverte pluridisciplinaire HAL, est destinée au dépôt et à la diffusion de documents scientifiques de niveau recherche, publiés ou non, émanant des établissements d'enseignement et de recherche français ou étrangers, des laboratoires publics ou privés. 


\title{
Dimensioning a product in preliminary design through different exploration techniques
}

\author{
Bernard Yannou ${ }^{1,{ }^{*}}$, Nadège Troussier $^{2}$, Alaa \\ Chateauneuf $^{3}$, Nassim Boudaoud ${ }^{2}$, Dominique \\ Scaravetti ${ }^{4}$ \\ ${ }^{1}$ Laboratoire Genie Industriel, Ecole Centrale Paris \\ Grande Voie des Vignes, 92290 Chatenay-Malabry, France \\ E-mail: bernard.yannou@ecp.fr \\ *Corresponding author \\ ${ }^{2}$ Laboratoire ODIC \\ Université de Technologie de Compiègne \\ Compiègne, France \\ nadege.troussier@utc.fr; nassim.boudaoud@utc.fr
}

${ }^{3}$ LGC

Polytech'Clermont-Ferrand

Université Blaise Pascal

Clermont-Ferrand, France

alaa.chateauneuf@polytech.univ-bpclermont.fr

${ }^{4}$ Laboratoire TREFLE

UMR CNRS 8508

Arts et Métiers ParisTech

Talence, France

dominique.scaravetti@bordeaux.ensam.fr

\begin{abstract}
Once a design concept chosen and parameterized, the embodiment design stage consists in choosing materials and dimensions to ensure a "good matching" with the expected performances. In this context of the first stages of a parametric design, several approaches exist which correspond to slightly different complexities and issues, which are more or less easy to implement and must, consequently, be used at different stages. We consider in this paper three families of approaches: (1) exploring design (parametric) dimensioning under uncertainty (through constraint programming techniques, representations of feasible design points or Pareto frontiers), (2) robust design and multidisciplinary optimization and, lastly, (3) design for reliability. One advocates and states in this paper that these approaches must be used in that order of increasing complexity. Indeed, applying an approach allows to quickly figure out inadequacies with performance specifications or initial allowable bounds of design parameters and then to backtrack or to refine the design issue before passing to the next stage or approach. We illustrate that phenomenon in successively applying the three approaches on an ever more complex parametric dimensioning issue of a two-member truss structure. We clearly show that the successive optimal designs obtained by the three categories of methods are notably different, but that the optimal point obtained in a given approach is used to explore its surroundings in the next approach.
\end{abstract}




\section{B. Yannou, N. Troussier, A. Chateauneuf, N. Boudaoud, D. Scaravetti}

Keywords: design exploration; robust design; design for reliability; constraint programming; Pareto solutions; embodiment design, parametric design, design optimization.

Reference to this paper should be made as follows: Yannou, B.; Troussier N.; Chateauneuf A.; Boudaoud N.; Scaravetti D. (xxxx) 'Dimensioning a product in preliminary design through different exploration techniques', Int. J. Product Development, Vol. X, No. Y, pp.000-000.

Biographical notes: Bernard Yannou is a Full Professor of Industrial and Mechanical Engineering at the Laboratoire Génie Industriel of Ecole Centrale of Paris, France. He received a M. Sc. (1988) in Mechanical Engineering from Ecole Normale Supérieure of Cachan, and M. Sc. (1989) in Computer Science from Paris-6 University. He received a Ph. D. (1994) in Industrial Engineering from Ecole Normale Supérieure of Cachan. His research interests are centred on the preliminary stages of product design: defining the design requirements, synthesizing product concepts, rapid evaluation of product performances, preference aggregation of the product and the project performances for the supervision of the design process, subjective and perceptual evaluation of products.

Nadège Troussier is an Associate Professor of the University of Technology of Compiègne, France. She obtained at the Grenoble Institute of Technology, France, a Master of Science in Mechanical Engineering and a Mechanical Engineering Degree in 1996, and a Ph.D. in Mechanical Engineering in 1999. Her research interests concern Product Design and, more accurately, the integration of virtual prototyping in product design process. Within this framework, she contributes to a knowledge management and knowledge modeling approach in order to enhance the use of structural analysis as soon as possible in the design process in order to improve product and process robustness.

Alaa Chateauneuf is a Full Professor in Structural Design, Optimization and Reliability, at Blaise Pascal University, Clermont-Ferrand, France. He received a M. Sc. (1989) in Civil Engineering from the INSA of Lyon and a Ph. D. (1993) in Engineering science from Blaise Pascal University. His research activities are focused on Structural Reliability and Optimization, through the development of original methods and tools for Non-linear modeling, Optimal design and Reliability assessment, with applications to offshore platforms, reinforced concrete structures, railways and automotive industries. He has supervised more than $20 \mathrm{Ph}$. D. theses in civil and mechanical engineering. He is the author and co-author of six books in design, optimization and reliability, as well as two reliability-based design software.

Nassim Boudaoud is an Associate Professor of industrial engineering of the University of Technology of Compiègne, France. He received a M. Sc (1994) in Control Engineering from the UTC. He received a Ph. D. (1997) in Control Engineering from the UTC. His research topics concern statistical analysis for quality management, design of experiments, robust design and design for sixsigma.

Dominique Scaravetti is an Associate Professor at the TREFLE Laboratory of the Arts et Métiers ParisTech Graduate School of Engineering since 2006. He received a Ph. D. (2004) in Mechanical Engineering from ENSAM. Before that, he had been teaching at ENSAM since 1997. His research interests are centred on decision aid during preliminary design phases. 


\section{Introduction and overview of embodiment design methods}

The exploration of design parametric dimensioning under uncertainty can be made in using (1) fuzzy sets theory applied to design engineering (Antonsson and Otto, 1995), (2) Monte Carlo simulations (i.e. generating feasible design points) followed by an exploration within the feasible design space (see for example (Stump et al., 2004)) and (3) constraint programming techniques (Yannou and Harmel, 2005). We have already experimented that constraint programming techniques may be convenient (as soon as they are well tuned) to quickly result in an encompassing approximation of the design space (see (Yannou and Hamdi, 2004)). In addition, a further Monte Carlo sampling within this approximate space has been shown (see (Yannou et al., 2005)) to be very efficient to straightforwardly obtain a good ratio of feasible design points (i.e. respecting the different design constraints and specifications). An exploration of a set of feasible design points is a valuable alternative and even may be preferred to the use of a global objective function (or a global preference aggregation function) because in preliminary design the weighting of the different objectives may be very subjective. This is why we believe that exploration lets the designer to be more opportunistic and even to acquire a better comprehension of the potential of its parameterized design. Lastly, techniques for generating and exploring the Pareto optimal solutions are evoked. On the example of the truss structure, it is shown that the design issue is already well constrained and that a tradeoff is useful to loosen somewhat some specifications in order to keep a sufficient degree of freedom to tackle robust design and design for reliability.

The multi-disciplinary design uses multi-objective optimization algorithms to find the best design that fulfills the numerous performances to be reached. These performances are evaluated using numerous disciplinary theories. It is often difficult to take the different theories into account in the unique formalism of an optimization approach. We propose to use a metamodel (or surrogate model) (Papalambros, 2002) that enables to formalize multi-disciplinary knowledge and to evaluate several performances using just one kind of mathematical formulation. To illustrate the elaboration of a metamodel, the design of experiments method and an identification method (least mean square method) are used to identify predefined mathematical functions that link design parameters to performances.

Once these functions are validated, they represent with a unique form several physical phenomena and can be used to evaluate, approximately but very quickly, the behavior of the design that can be evaluated. This evaluation can be made according to different criteria such as robustness or reliability.

As defined by Taguchi, a robust design is a design of a product so that its functionality varies minimally despite of disturbing factor influences, which can be associated to environment factors, usage factors or technical factors such as design parameters... The aim is not to find the most performing design according to the set of performances to be reached, but to guarantee that the higher level of performing is guaranteed whatever the perturbations on the definition, process, usage or environment parameters are. The use of desirability functions to formalize the optimization goal and the signal/noise ratio in design of experiments enables to find on the truss structure the most robust solution. 


\section{B. Yannou, N. Troussier, A. Chateauneuf, N. Boudaoud, D. Scaravetti}

The design for reliability aims to find the optimal solution that fulfills a given reliability condition. The fluctuation of loads, the variability of material properties and the uncertainties regarding the analysis models, contribute to make the performance of the optimal design different from the expected one. In this sense, the optimization process has a large effect on the structural safety and/or reliability. However, the safety factor approach cannot ensure the required safety level, as they do not explicitly consider the probability of failure regarding some performance criteria. In other words, the optimal design resulting from deterministic optimization procedures does not necessarily ensure the required reliability level. The design for reliability allows us to consider the safety margin evolution, leading to the settlement of the best compromise between the life-cycle cost and the required reliability. This task is further complicated due to the inherent nondeterministic nature of the input information. For this reason, many analysis methods have been developed to deal with the statistical nature of data. The process efficiency is mandatory to deal with realistic engineering problems (Kharmanda et al., 2002); the metamodels can thus be very helpful in achieving the reliability-based optimal design with a reasonable computation effort. The solution obtained on the reliability basis is rather robust as the uncertain parameters are penalized during the design process, compared to a greater commitment of the well-controlled parameters. Practically, the design problem is formulated as a minimization of the cost function under some prescribed reliability targets (Aoues and Chateauneuf, 2007, in Press).

For the robust design and design for reliability approaches, the truss structure example has been made more complex in adding two performances: fundamental frequency and section area.

This paper can be considered as a brief survey of the main approaches that can be followed during the embodiment design stage of a product. Its purpose is, on a practical example of the parametric dimensioning of a truss structure, to concurrently apply these approaches so as to figure out that it is worthy to apply them successively in an ever refining embodiment design process. The paper is structured into five more sections: a presentation of the truss structure dimensioning issue, one section for each of the three approaches and a section of concluding remarks.

\section{The truss structure dimensioning design issue: A first modelling}

Our case study consists of dimensioning the two members of the truss structure shown in Figure 1. This problem was originally proposed by Wood, et al. (Wood et al., 1989) to compute imprecise performance parameters from imprecise design parameters via fuzzy sets theory. This example has also been used by Scott, et al. (Scott and Antonsson, 2000) in a different parameterized form to select an optimal Pareto solution that could not be selected via a linear aggregation function using importance weights. For this example, we use the exact parameterization and initial design variable ranges of the truss structure described by Wood, et al. (Wood et al., 1989), but we have chosen the more complex design constraints and performance parameters used by Scott, et al. (Scott and Antonsson, 2000). 
Dimensioning a product in preliminary design

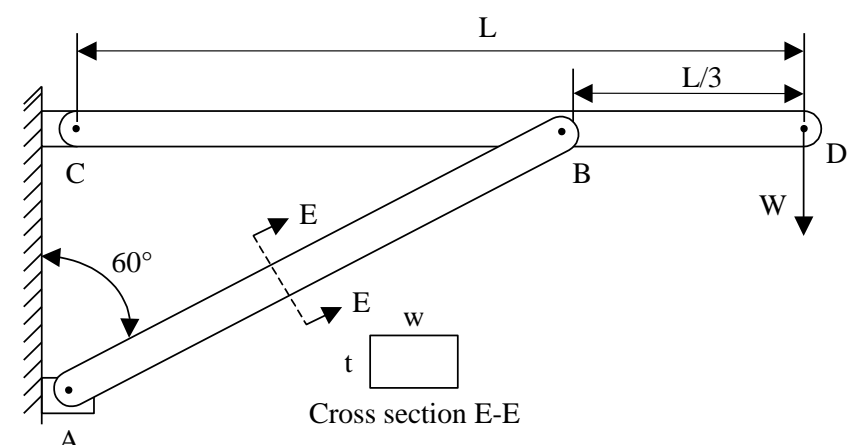

Figure 1 The parameterization of the truss structure

The requirement is to design a mechanical structure supporting an overhanging vertical load at a distance $L$ from the wall with a minimal mass. One possible configuration (see Figure 1) consists in a two-member pin-jointed bracket with a horizontal member $(C D)$ and a compression member $(A B)$ attached to the wall at an angle of sixty degrees. The common pin is located at two thirds of $L$ from the wall. Both members have rectangular cross sections: $w_{A B} \times t$ for $(A B)$ and $w_{C D} \times t$ for $(C D), w$ standing for width and $t$ for thickness. Additional design decisions have been made: the material of both members is steel, and we impose $w_{C D}=w_{A B}-0.025$. The designer has to make decisions for the values of the following design parameters: $t, w_{A B}$ and $L$. Moreover, the specification of the overhanging load $W$ is imprecise at the beginning of the design process, varying from $15-20 \mathrm{kN}$; consequently, $W$ is treated as a fourth design variable.

The two mechanical constraints to satisfy are:

- the maximum bending stress, $\sigma_{b}$, in member $(C D)$ must be less than or equal to the allowable bending limit, $\sigma_{r}$ (here $225 \mathrm{MPa}$ for steel).

- $\quad$ the compression force $F_{A B}$ in member $(A B)$ must be less then or equal to the buckling limit $F_{b}$.

The maximum bending stress, $\sigma_{b}$, is located at point $\mathrm{B}$ and is given by the following formulas involving $W_{C D}$, the weight of member $(C D)$ :

$$
\sigma_{b}=\frac{2 L\left(W+\frac{W_{C D}}{6}\right)}{w_{C D} t^{2}} \text { with }\left\{\begin{array}{c}
W_{C D}=\rho g w_{C D} t L \\
w_{C D}=w_{A B}-0.025
\end{array}\right.
$$

The compression force in member $(A B)$ is given by the following formulas involving $W_{A B}$, the weight of member $(A B)$ :

$$
\begin{aligned}
& F_{A B}=\sqrt{\left\{\frac{9}{2 \sqrt{3}}\left(W+\frac{W_{C D}}{2}+\frac{W_{A B}}{3}\right)\right\}^{2}+\left\{\frac{3}{2}\left(W+\frac{W_{C D}}{2}\right)\right\}^{2}} \\
& \text { with } W_{A B}=\rho g w_{A B} t L_{A B} \text { and } L_{A B}=\frac{4 \sqrt{3}}{9} L
\end{aligned}
$$


The buckling limit in member $(A B)$ is given as:

$$
F_{b}=\frac{\pi^{2} E I_{A B}}{L_{A B}^{2}}=\frac{9 \pi^{2} E w_{A B} t^{3}}{64 L^{2}}
$$

The performance variables are the mass $M$ of the structure (to be minimized) and the safety factor, $s$, i.e., the amount of over-dimensioning beyond the satisfaction of the two mechanical constraints. The mass $M$ is given by:

$$
M=W_{A B}+W_{C D}
$$

The safety factor of the truss structure $s$ is the minimum between the safety factor below the allowable bending limit, $\sigma_{b}$, namely, $s_{\sigma}$, and the safety factor below the buckling limit, $F_{b}$, namely, $s_{F}$, which is expressed as:

$$
s_{\sigma}=\frac{\sigma_{r}}{\sigma_{b}}, \quad s_{F}=\frac{F_{b}}{F_{A B}}, \quad s=\min \varsigma_{\sigma}, s_{F}-
$$

The two mechanical constraints may be merely expressed by: $s_{\sigma} \geq 1, \quad s_{F} \geq 1$ or simply by the single constraint:

$$
s \geq 1
$$

\section{Exploration of design parametric dimensioning under uncertainty}

Design space exploration during embodiment design is an active research field. It consists in exploring the relationships between the choices of design parameter values and the performance variable values. This exploration provides the designer with a deep understanding of the potential of the given design concept that is studied, in comparison of a direct optimization of an objective function (function of the performance values). Often, a design space exploration is performed within a "design under uncertainty" process which is a process of dimensioning the product in progressively and consciously narrowing the domains (of allowable values) of design parameters while respecting the specifications on performance variables and constraints (like the aforementioned mechanical constraints). Three family of techniques are used to support the uncertainty reduction forwardly (from parameter domains to performance domains) and backwardly (from performances to parameter domains):

- Fuzzy sets theory in design engineering (see (Antonsson and Otto, 1995), not detailed here),

- Probabilistic techniques: most of these approaches consist in generating a number of feasible design points (complying with the constraints) and apply graphical postprocessing to visualize correlations between variables, the Pareto frontier or a preference structure among the design solutions (see (Stump et al., 2004)). The generation of feasible design points is often a statistical (Monte Carlo) generation of potential candidates sampled within initial variable domains, followed by the checking of constraints, which may become inefficient if the design problem is 


\section{Dimensioning a product in preliminary design}

highly constrained since a majority of candidates that are generated do not belong to the (small) feasible solution space.

- Constraint Programming (CP) techniques. They are not only well adapted to a numerical exploration of dimensional values for both performance variables and design parameters. In addition, they are also adapted to topological explorations of design concepts or architectures under constraints and, by the way, to the consideration (and exploration) different configurations of system functioning constraints (such as external load conditions) depending of the considered lifecycle stages (like flight phases, in case of (Scaravetti et al., 2006b)). Scaravetti et al (Scaravetti et al., 2006a) have studied the way that CP techniques modelled under the CSP formalism (Constraint Satisfaction Problem, which is generally the case for CP techniques) must be used within an industrial deployment of a design project: which constrained variables, which constraints, evaluation of several alternatives, choice within component catalogs).

With Constraint Programming (CP) over reals, performance variables and design parameters are modelled as intervals of allowable values. These constrained variables may be equated to uniform distributions of values in probabilistic modelling. CP techniques consist of sophisticated evolutions of interval analysis or interval arithmetics (see (Moore, 1979)) applied on a set of analytical constraints. Starting from a set of initial domains for the constrained variables and from a set of mathematical constraints linking the variables, different CP consistency or filtering techniques (such as Hull, Box, weak$3 B$ or $3 B$, see for instance (Benhamou et al., 1999) and (Yannou and Harmel, 2005)) try to contract as much as their consistency degree allows the variable domains so as to eliminate infeasible values. This domain contraction stage is called the filtering stage. One tries to result in the most tightened Cartesian product of intervals, ensuring at any moment that any feasible solution is kept inside. This last important property refers to the completeness property and guarantees that the contraction process results in an outer design space approximation.

In the second stage, the mechanism of domain splitting (bisection for instance) is recursively applied in parallel with the filtering mechanism. A search tree is built until a stopping criterion (e.g., width of the domains, number of solutions) is reached. This branch-and-prune algorithm allows pruning out large parts of the design space whenever a domain is found to be empty. At the end of the process, the design space is approximated by a number of elementary and disjointed Cartesian products of small intervals, denoted as boxes. The resulting hull of boxes provides the designer with valuable information about the potential values remaining for any design variable at this stage. Finally, a graphical representation of this collection of $n$-dimensional boxes ( $n$ being the number of constrained design variables) is easy and convenient for obtaining good pictures of the resulting design space (by 2D or 3D projections on pairs or triplets of design variables).

Figure 2 illustrates the four outer approximations of the design space that we can consider in a $\mathrm{CP}$ computation process, namely:

- The initial domains;

- The filtered domains after the uncertainty reduction propagation has been made for the first time; 


\section{B. Yannou, N. Troussier, A. Chateauneuf, N. Boudaoud, D. Scaravetti}

- The hull of boxes, i.e., the projection on variable domains of the collection of boxes that have not been considered inconsistent after the domain splitting process (with no guarantee of any actual solution inside); and

- The collection of boxes itself.

It is obvious that, these four outer approximations of the design space are ordered in an increasing rank of refinement.

We have already showed in (Yannou and Hamdi, 2004) that the graphical representations of the collections of boxes could be meaningful for the designer(s) to perform relevant analyses of variable correlations and tendencies and making good decisions in a multi-stages "design under uncertainty" process. This is a first important utility of CP techniques in preliminary parametric dimensioning. Two stages of such a dimensioning process are illustrated in Table 1 with two cases:

- - The case of a "not so constrained" design problem, which means that the initial domain is not large compared to the effective solution space. This is the situation of Case \#1 of the specification constraints on the truss structure (the safety factor is just constrained to be greater or equal than 1).

- - The case of a "highly constrained" design problem, which means that the initial domains are much larger than the actual solution space. This is the case of Case \#2 of the specification constraints on the truss structure.

Note that in both cases, the ever more constrained problem is performed throughout constraints on performances. It means that the designer(s) can, here, really design in a functional manner, starting from the need and propagating consequences towards the solution (the means). This interesting facility is here permitted by the back-propagation properties of the $\mathrm{CP}$ filtering mechanisms.

\begin{tabular}{|c|c|c|}
\hline $\begin{array}{l}\text { Design } \\
\text { parameters }\end{array}$ & $\begin{array}{l}\text { Performanc } \\
\text { e variables }\end{array}$ & Constants \\
\hline$t \in \mathbf{X} .04,0.10^{-}$ & $M \in \backslash,+\infty$ & $E=207 \cdot 10^{9} \mathrm{~Pa}$ \\
\hline$w_{A B} \in \mathbf{X} .04,0.13^{-}$ & & $\rho=7830 \mathrm{~kg} / \mathrm{m}^{3}$ \\
\hline$L \in\left\lfloor, 4_{-}^{-}\right.$ & $s_{F} \in \mathbf{\prod},+\infty$ & $g=9.81 \mathrm{~m} / \mathrm{s}^{2}$ \\
\hline$W \in\lfloor 5000,20000$ & $s \in \mathbf{I},+\infty$ & $\sigma_{r}=225 \cdot 10^{6} \mathrm{~Pa}$ \\
\hline
\end{tabular}


Dimensioning a product in preliminary design

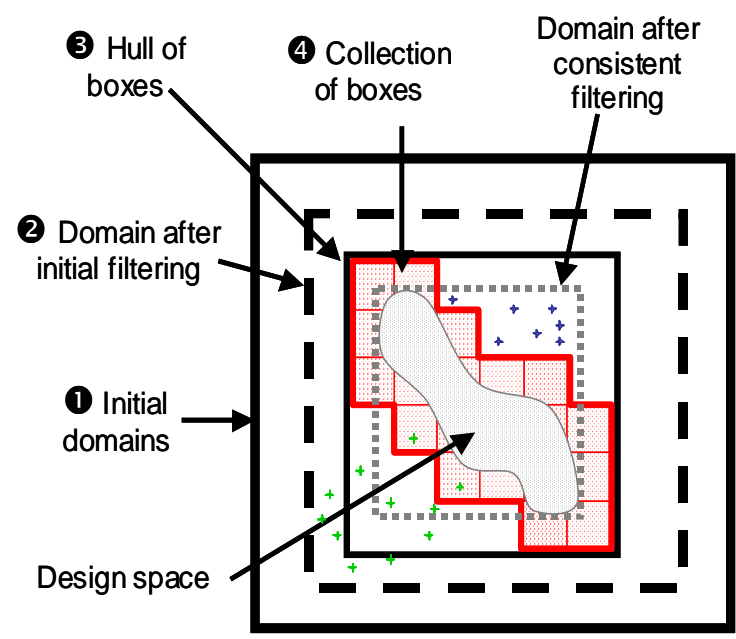

Figure 2 Initial interval domains of design parameters and performance variables for the trussstructure problem (left). The four successive outer approximations of the design space available after a Constraint Programming (CP) computation (right).

Another interesting case of domain reductions by $\mathrm{CP}$ techniques may be seen on the design exploration of an aircraft air conditioning system by Scaravetti et al (Scaravetti et al., 2006b). The design problem consists in optimizing the internal structures of the heat exchangers while satisfying the functioning constraints imposed by the system environment. The design variables are geometric and structural variables (lengths, surface types and pass number in the exchangers) but also thermodynamic variables (pressures, mass flow rates, temperatures). The performance variables are linked to efficiencies, mass, drag induced.

After having sufficiently explored the design space of our truss structure and after several domain (and uncertainty) reductions, the designer(s) has converged toward a small design space of interest. It is time now to use probabilistic techniques to result in a cloud of feasible design points that one could apprehend one by one. We can perform a brute Monte Carlo simulation in sampling 100,000 design points by random trials within the initial domains of the design parameters (provided in Figure 2.left). After the checking of mechanical constraints, we have obtained (see also (Yannou et al., 2005)) 15,000 feasible design points in the "not so constrained" case (case \#1) versus only 4 in the "highly constrained" case (case \#2). These feasible and unfeasible design points have been represented in Figure3-left to figure out the very low ratio of feasible design points. It is then problematic when the designer wants to finely explore the design space since the design space is not dense enough to display a continuous variation of performances. A second utility of CP computation techniques has then been proposed by Yannou et al in (Yannou et al., 2005): the ratio of feasible design solutions is much more efficient when sampling inside the collection of boxes (obtained from a primary CP computation). In that case \#2, the number of feasible design points has been 9,500 instead of 4 (Yannou et al., 2005), a great gain in efficiency. 
Table 1 CP computation of the truss structure - considering two series of specification constraints

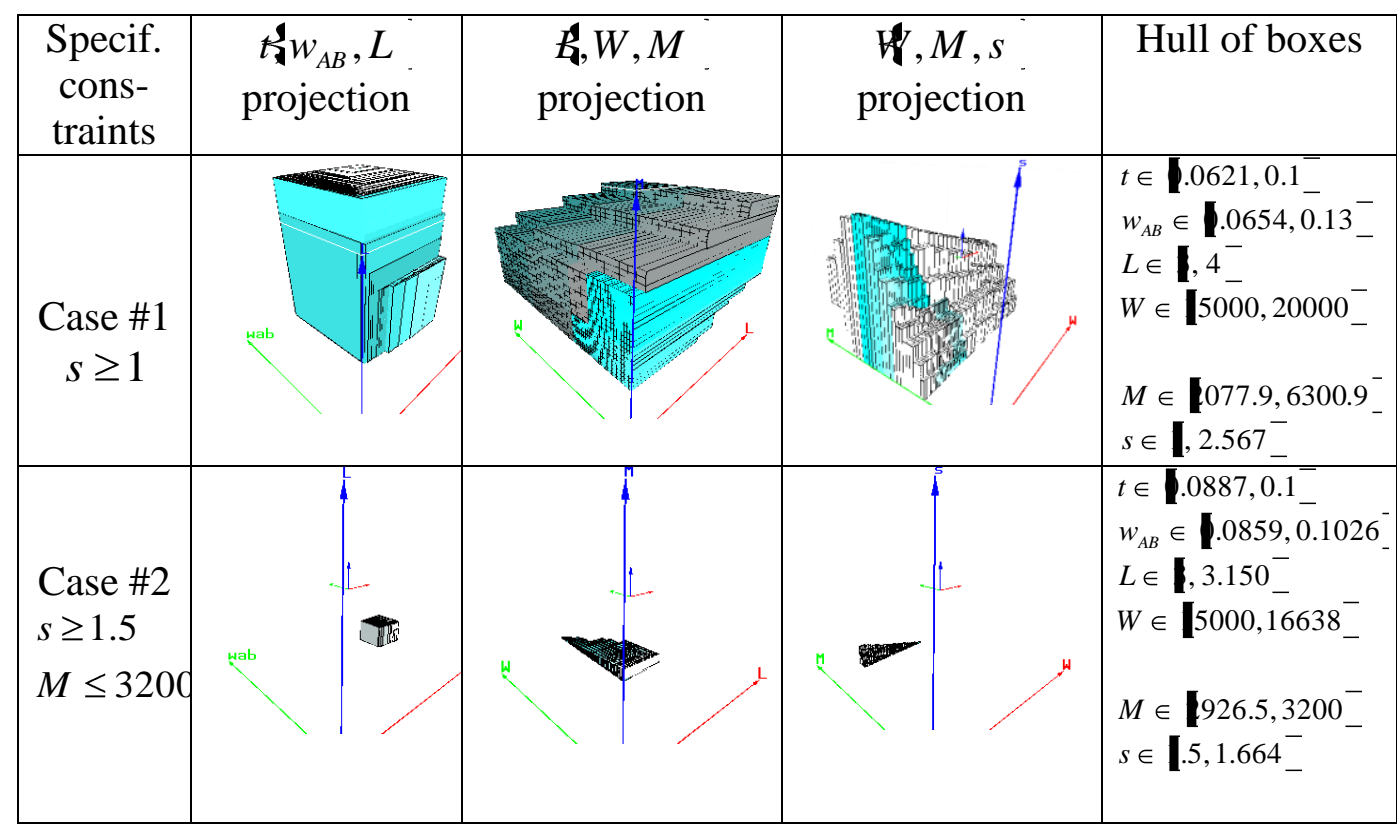

Only a subset of the feasible design points may be eligible as the best or preferred solution: this is the set of Pareto optimal solutions. This set of Pareto solutions is represented as a curve in the performance space: this is the Pareto frontier. By definition, a Pareto frontier (see (Messac et al., 2003)) is the locus of non-dominated solutions, a dominated solution being a solution (design point) for which at least one solution (design point) is better for any of the performances. Highlighting the subset of Pareto solutions among the feasible design points is then very meaningful for designers. In (Stump et al., 2004) and in (Scaravetti et al., 2006b), the authors have developed graphical interactive tools in informing the designer on the performance values of a clicked optimal Pareto solution (the click is made in a performance plane or space) and in highlighting the corresponding design parameter values (made in a -design-parameter plane or space). 
Dimensioning a product in preliminary design

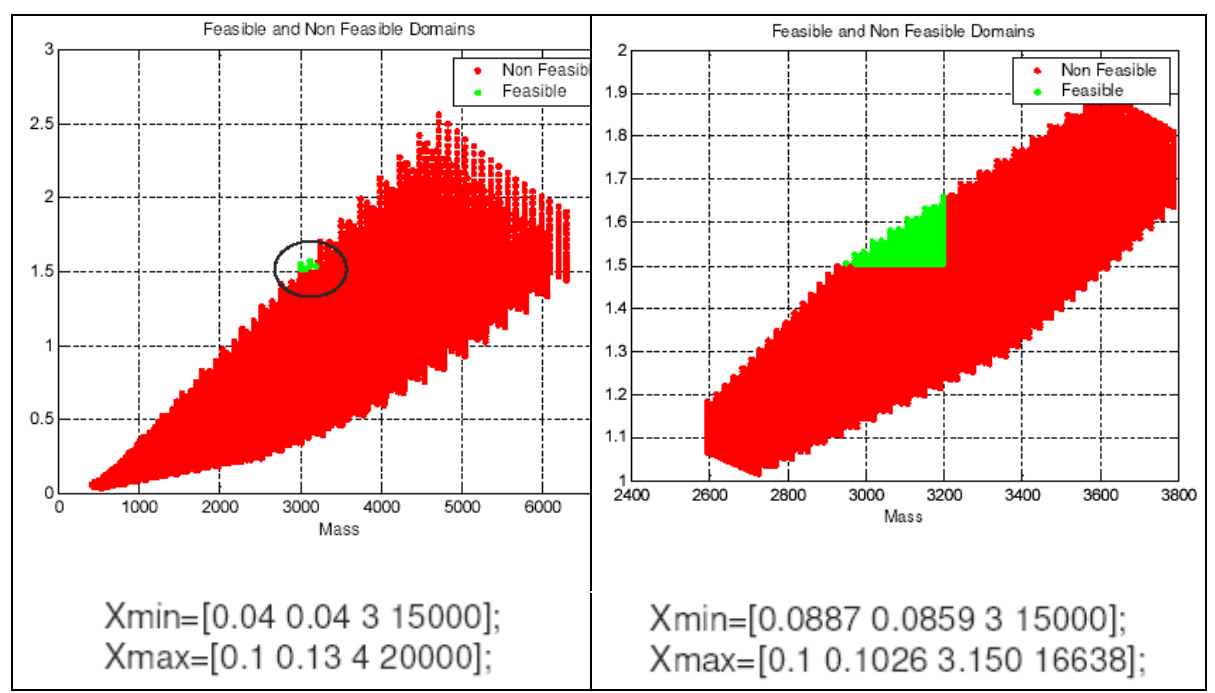

Figure 3 Representation of feasible and unfeasible design points in the performance space for case \#2 with a sampling of 100,000 design points within two different sets of domains $\mathrm{X}=[\mathrm{t}, \mathrm{w}, \mathrm{L}, \mathrm{W}]$.

At this stage, it would be useful to benefit from a procedure to choose one of the Pareto optimal solutions as the preferred solution. Here, optimization techniques are often used. An objective function must then be built as a function of the performances (here $M$ and $s$ ). A traditional form of this objective function is given by the following formula:

$$
\alpha \frac{M-M_{\text {min }}}{M_{\text {max }}-M_{\text {min }}}+\left(-\alpha \frac{s_{\text {max }}-s}{s_{\text {max }}-s_{\text {min }}} \text { with } \alpha \in \mathbf{Q}, 1_{-}^{-}\right.
$$

The weighting factor $\alpha$ must be identified by the designers. It is well known that, in case of a convex Pareto frontier, making varying $\alpha$ results in running all along the Pareto frontier (see Figure 4). But, in case of a concave Pareto frontier, some portions are not covered by an optimization process and, consequently, some Pareto solutions practically become non-eligible. This non trivial issue may be solved in using more sophisticated forms of the objective functions (see Scott and al for instance (Scott and Antonsson, 2000)). 

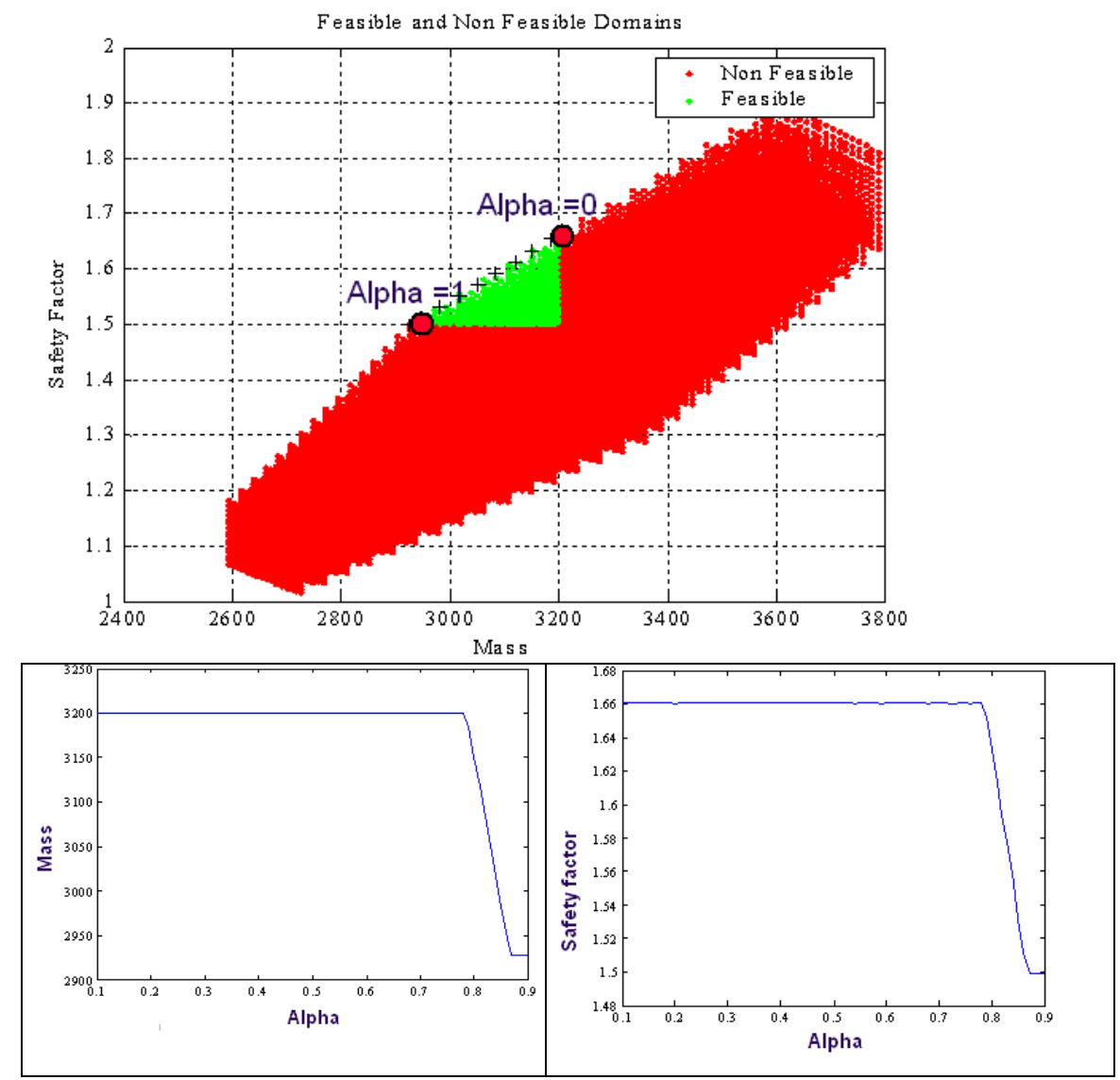

Figure 4 An optimization of an objective function (function of $s$ and $M$ ), making varying the $\alpha$ parameter of linear combination, describes the all Pareto frontier.

\section{Robust design}

The aim of this section is to use a robust design approach as described by Taguchi (Fowlkes and Creveling, 1995) on the truss example. The first section has presented how to explore the design space and to identify the space of decision among the values of the different design parameters in order to reach fixed specifications or performances. Then, knowing the ranges of parameters that enable to reach the specifications, several criteria can be formalized to focus not only on a convenient design, but also on the best design. The criteria that we propose to use is the robustness, i.e. we search for the design solution that guaranties the level of performances whatever the variabilities on the design parameters are. That is to say that, the design parameters are chosen in order to have the performance levels the less sensitive as possible to the variabilities of design parameters, process variables and environment factors. These uncontrolled variabilities (for instance due to tolerances) should affect the less as possible the levels of performances. The 


\section{Dimensioning a product in preliminary design}

problem is not to suppress or control the variabilities but to minimize their effects on performances.

Two different scales of design parameter values are used. The first level (concerned with the first section of this paper) of the scale is dealing with the variation of design parameters (different values of each design parameters that should be chosen to define the design). The second level is concerning with the variabilities around a design parameter value. These variabilities are small compared to the possible variation of the design parameter values but it is very important to be able to take these variabilities into account for decision making in the whole design process (Crossland et al., 2003, Ullman and D'ambrosio, 1995).

In the present section, we will take some design parameter values, identified for the truss example in the first part of the paper as convenient values, to reach the specifications in terms of performances. Around these values some variabilities are considered to take into account some uncertainties on design parameters, process variables and environment factors. Then, a robust design approach is used to propose the most robust design in the design space under uncertainties.

\subsection{Specificities of the truss example for robust design}

Managing the quality of the truss design requires ensuring the reachable performances levels in a constrained and uncertain context. Even if a common practice is to find the first convenient design that enables to reach the functional specifications, numerous research works are conducted to improve the design methodologies in order to be able to find the best design instead of a good design. N. Suh with Axiomatic Design (Suh, 2001) is one of the first authors who proposes an approach that enables to guide design activities based on two axioms that evaluate the design all along the design process. Quality Function Deployment is especially appropriated to evaluate the quality of the product all along the design process. However, we will use the vocabulary introduced by the informational spaces proposed by N. Suh (in the following description of the robust design approach. Designing is then a transformation between the variables through these different spaces. Even if the role of history and knowledge is not highlighted by this model, it will be used to formalize the truss problem in order to use a robustness criterion for its design evaluation (El-Haik, 2005).

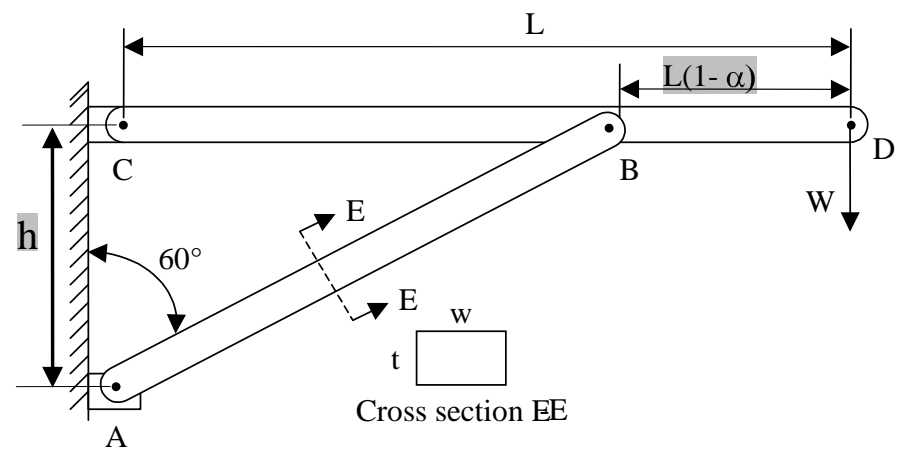

Figure 5 The new parameters of the truss structure 


\section{B. Yannou, N. Troussier, A. Chateauneuf, N. Boudaoud, D. Scaravetti}

On the truss structure the performances or functional requirements initially considered are $M, s_{\sigma}, s_{F}, s$ and the design parameters are $t, w_{A B}, L, W$ as described in Figure 5. Other design parameters are added in the problem formulation in order to have more sensitive performances. The two more design parameters that we consider in this section are: $h$, the distance between the top and the bottom of the truss, and $\alpha$ that parameterizes the position of the joint linking the two members of the truss along the horizontal member.

\subsection{The Taguchi robust design approach}

Axiomatic Design provides two axioms (the independence and minimal information axioms) that enable to evaluate the quality of the design (Suh, 2001). If these axioms present a great interest for new innovative design on "uncoupled" systems, this design context is not usual when complex products are improved from a design to another one and the constraints imply that the axioms cannot be respected in practice. For instance, on the previous truss design formulation, the two axioms are not respected by the formulation used in engineering design. Then, under specific conditions, a multiobjective optimization formulation can be formulated in order to find the solution that provides the best level of performances. However, the design solution obtained is often sensitive to design parameters variabilities on design parameters due to the process (uncertainties managed as tolerances), the environment (uncertainties not managed but to support such as temperature, humidity, etc) but also due to desired variabilities such as users preferences, contexts of use of the product, etc. For instance the best solution for the truss structure previously defined is very sensitive to several variabilities such as illustrated in Figure 6. In this figure, the performance $s$ is provided with respect of the $h$ and $t$ design parameters.

In this case, the robust design approach enables us to find the best solution and the most robust to design parameters variabilities. Robust design has been introduced by G. Taguchi and is defined in (Flowkes and Creveling, 1995). The usual design approach aims at finding a design solution that reaches functional specifications and minimizing the uncertainties on design parameters. If some design parameters can be defined accurately, some tolerances always exit and perturbing factors can always be considered. Then, the robust design approach proposes to take into account the variabilities in the performances evaluation instead of avoiding it. Taguchi's proposition is based on the design of experiment (DoE) method to evaluate the sensitivity of the performances taking uncertainties into account. These uncertainties or variabilities are introduced as noise factors in the Taguchi table of the design of experiment as a small variability around each value of the design parameters. The impact of these variabilities around the design parameters values can then be analyzed and the most robust solution can be obtained by maximizing the signal to noise ratio. 


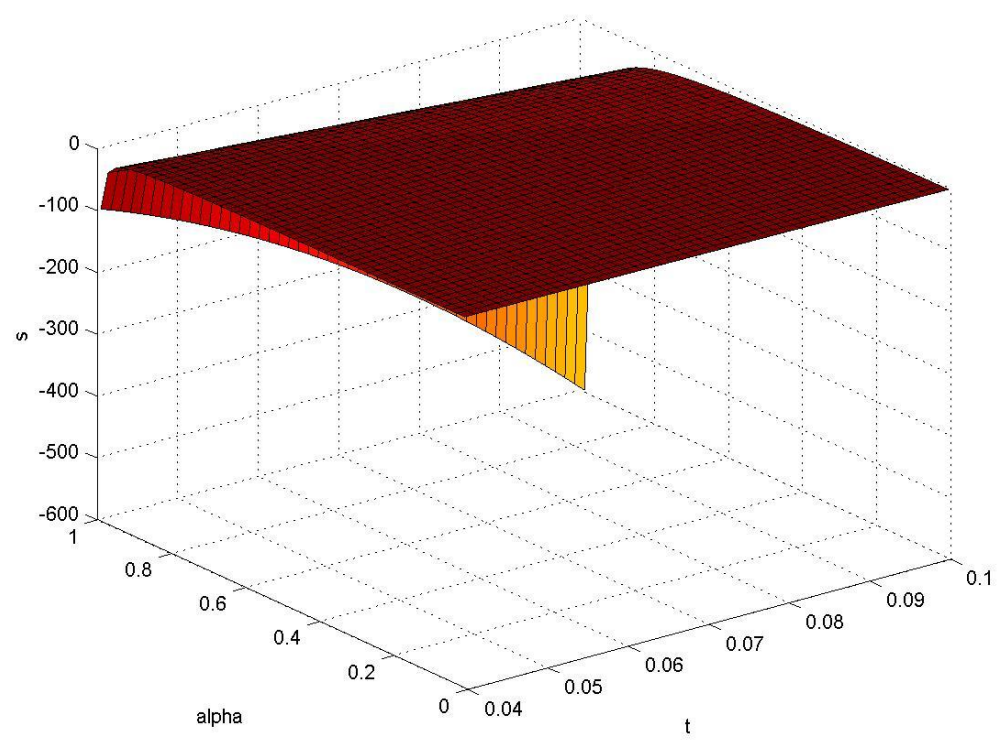

Figure 6 An example of a response surface which provides s, one of the performances $\{$ FRs $\}$ with respect to some sensitive design parameters of $\{D P s\}, t$ and $\alpha$

The design of experiment considered on the truss example is provided in Table 2. The mechanical laws are a bit more sophisticated than those formulated by formulas (1) to (6) and are not provided here for reasons of brevity. These laws are used to evaluate the truss performances and fulfill the performances evaluation (the two last columns of Table 2). For each line of the design of experiment, a set of design parameters values are considered and a level of variability around the fixed value is also provided.

Then the response surfaces (fitted metamodel) are built for each performance and the data of robustness level is added on the response surfaces as a color level. For instance, Figure 7 provides the representation of a performance (here $M$ and $s$ ) with respect to two design parameters ( $h$ and $t$ ) and taking into account the level of the signal to noise due to the variabilities on $\alpha$ (the higher the color on the surface response, the higher the signal to ratio). 
Table 2 The first lines of the table of experiments used to identify a linear model with interactions

\begin{tabular}{|c|c|c|c|c|r|r|r|}
\hline$t$ & $w a b$ & $L$ & $W$ & $h$ & pise on & $M$ & \multicolumn{1}{|c|}{$s$} \\
\hline-1 & -1 & -1 & -1 & -1 & -1 & 142 & 141 \\
\hline-1 & -1 & -1 & -1 & -1 & 1 & 503,3 & 504,3 \\
\hline-1 & -1 & -1 & -1 & 1 & -1 & 507 & 506 \\
\hline-1 & -1 & -1 & -1 & 1 & 1 & 657,1 & 658,1 \\
\hline-1 & -1 & -1 & 1 & -1 & -1 & 142 & 141 \\
\hline-1 & -1 & -1 & 1 & -1 & 1 & 503,3 & 504,3 \\
\hline-1 & -1 & -1 & 1 & 1 & -1 & 507 & 506 \\
\hline-1 & -1 & -1 & 1 & 1 & 1 & 657,1 & 658,1 \\
\hline-1 & -1 & 1 & -1 & -1 & -1 & 189,3 & 188,3 \\
\hline-1 & -1 & 1 & -1 & -1 & 1 & 671 & 672 \\
\hline-1 & -1 & 1 & -1 & 1 & -1 & 553,1 & 552,1 \\
\hline-1 & -1 & 1 & -1 & 1 & 1 & 794,9 & 795,9 \\
\hline-1 & -1 & 1 & 1 & -1 & -1 & 189,3 & 188,3 \\
\hline-1 & -1 & 1 & 1 & -1 & 1 & 671 & 672 \\
\hline-1 & -1 & 1 & 1 & 1 & -1 & 553,1 & 552,1 \\
\hline-1 & -1 & 1 & 1 & 1 & 1 & 794,9 & 795,9 \\
\hline-1 & 1 & -1 & -1 & -1 & -1 & 979,8 & 978,8 \\
\hline-1 & 1 & -1 & -1 & -1 & 1 & 2154 & 2155 \\
\hline-1 & 1 & -1 & -1 & 1 & -1 & 2166 & 2165 \\
\hline-1 & 1 & -1 & -1 & 1 & 1 & 2654 & 2655 \\
\hline-1 & 1 & -1 & 1 & -1 & -1 & 979,8 & 978,8 \\
\hline 1 & 1 & 1 & 1 & 1 & 1 & $n\lrcorner-1$ & $n 1 r r$
\end{tabular}

Then, the most robust solution is found (given in Table 3 ) by maximizing the signal to noise ratio for the performances under consideration. The choice of the performance(s) to guaranty has to be done. In Table 2, we can find the most robust design solution considering the robustness of $s$ performance, taking the variabilities on $\alpha$ design parameter into account.

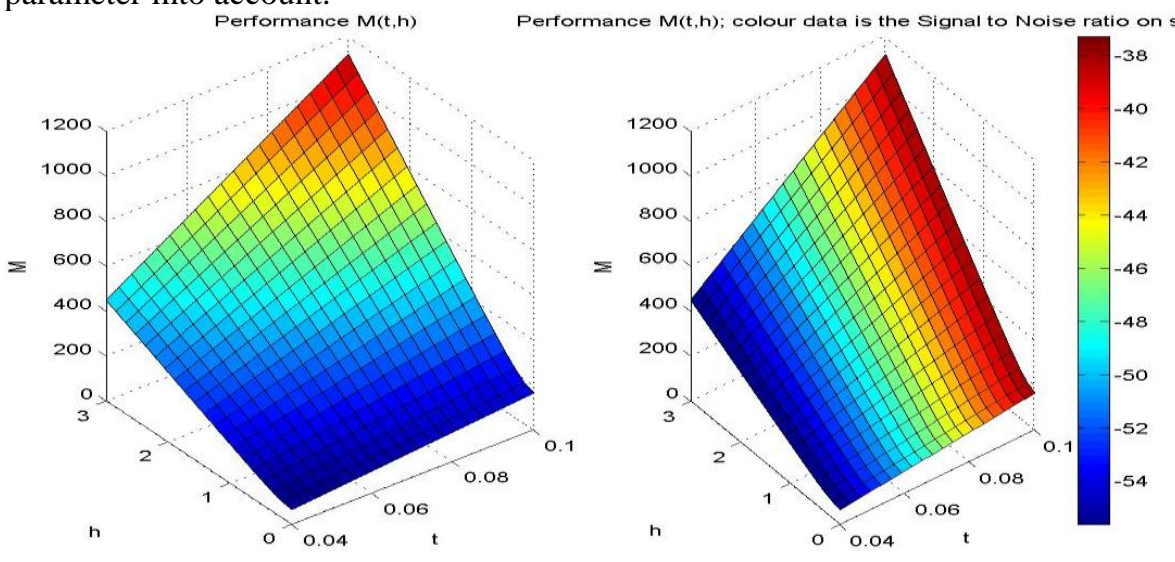


Dimensioning a product in preliminary design

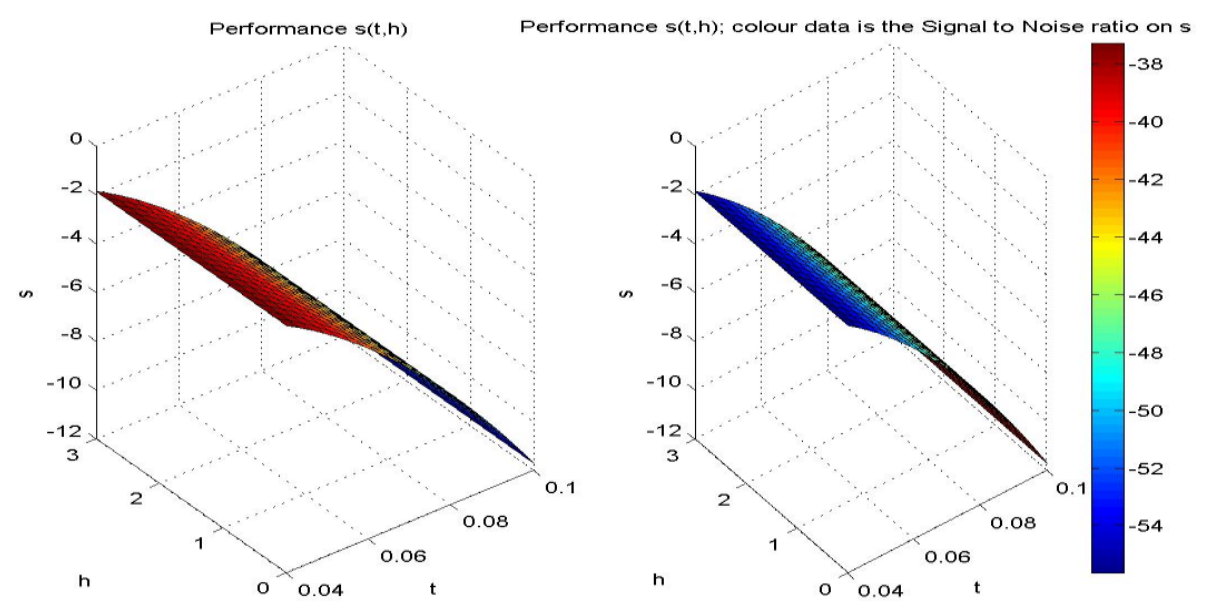

Figure 7 Example of a response surfaces on which robustness data are added (right part of figure)

Table 3 The best solution with the robustness criteria on s, with variabilities on $\alpha$

\begin{tabular}{|l|l|l|l|l|l|l|l|l|}
\hline & $t$ & $w_{A B}$ & $L$ & $W$ & $h$ & $\alpha$ & $M$ & $s$ \\
\hline $\begin{array}{l}\text { Robust } \\
\text { design }\end{array}$ & 0.1 & 0.13 & 3.42 & 17115 & 1.5 & 0.5 & & \\
\hline
\end{tabular}

If the most robust design solution is searched, then combination functions can be used, such as the desirability (or usability) functions to determine a global robustness. The most robust solution can be:

- the one that provides the less sensitive global desirability taking design parameters variabilities into account,

- the solution that maximizes the global desirability level (or minimizes a global loss function calculated on the whole responses), with a desirability level (or a loss level) associated to each signal to noise ratio provided by the impact of the variabilities on each performance.

\subsection{Results and interests of robust design for uncertainty management in embodiment design}

In order to make a decision under uncertainties in an engineering design context, and in particular in the embodiment design stage (defined in (Pahl and Beitz, 1996)), the robustness analysis can be used. The results provided, using a response surface with a color grid to evaluate the signal to noise ratio, enables the designer to choose not only the best design solution in the design space but also the less sensitive one to the design parameters variabilities due to uncertainties. The robustness can be studied performance by performance or considering a set of performances to guaranty. 


\section{B. Yannou, N. Troussier, A. Chateauneuf, N. Boudaoud, D. Scaravetti}

\section{Design for reliability}

The design of structures requires the verification of a certain number of rules resulting from the knowledge of physics and mechanical experience of designers and constructors. These rules come from the necessity to limit the loading effects such as stresses and displacements. Each rule represents an elementary event and the occurrence of several events leads to a failure scenario. In addition to the deterministic variables $d_{k}$ to be used in the system control and optimization, the uncertainties are modeled by stochastic variables affecting the failure scenario. The knowledge of these variables is not, at best, more than statistical information and we admit a representation in the form of random variables (noted $X_{i}$ whose realizations are $x_{i}$ ). For a given design rule, the basic random variables are defined by their probability distribution with some expected parameters.

\subsection{Reliability analysis}

The safety is defined as the state where the structure is able to fulfill all the functioning requirements: mechanical and serviceability, for which it is designed. To evaluate the failure probability with respect to a chosen failure scenario, a performance function $G\left(x_{i}, d_{k}\right)$ is defined by the condition of good operation of the structure. The limit between the state of failure $G\left(x_{i}, d_{k}\right) \leq 0$ and the state of safety $G\left(x_{i}, d_{k}\right)>0$ is known as the limit state surface $G\left(x_{i}, d_{k}\right)=0$ (see Figure 8 ).

In the First Order Reliability Method, the reliability level is defined by an invariant reliability index $\beta$, which is evaluated by solving the constrained optimization problem:

$$
\begin{aligned}
& \beta=\min V \boldsymbol{\bigotimes}_{i}\left(x_{j}\right) \overline{=} \sqrt{\sum_{i} \boldsymbol{\mho}_{i}\left(x_{j}\right)^{2}} \\
& \text { under the constraint }: G \bigotimes_{i}, d_{k} \leq 0
\end{aligned}
$$

where $V(\cdot)$ is the distance between the median point and the failure subspace in the normalized space and $T_{i}(\cdot)$ is an appropriate probabilistic transformation; the solution of this problem is called the design point. At the first order approximation, the failure probability $P_{f}$ is given as a function of the reliability index:

$$
P_{f}=\operatorname{Pr} \boldsymbol{F} \boldsymbol{\varangle}_{i}, d_{k} \leq 0_{-}^{-} \approx \Phi(-\beta)
$$

where $\operatorname{Pr}[$.$] is the probability operator and \Phi($.$) is the standard Gaussian cumulated$ function. 


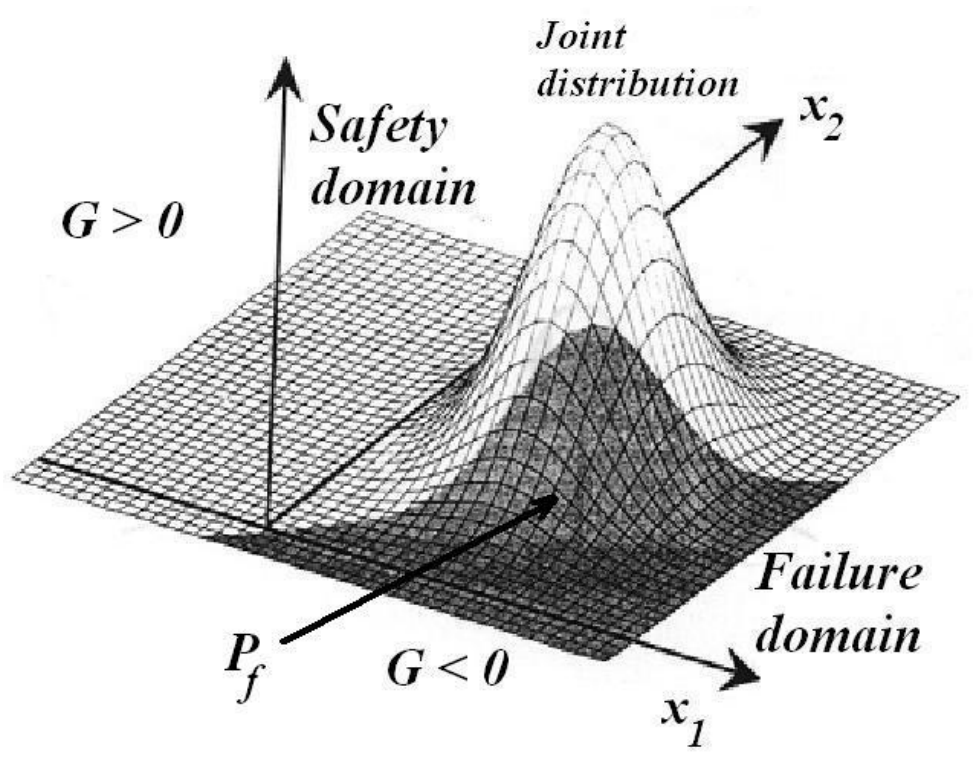

Figure 8 Parameter joint distribution and failure probability

\subsection{Reliability-based design optimization}

The Reliability-Based Design Optimization (RBDO) aims at searching for the best compromise between cost reduction and reliability assurance, by taking the system uncertainties into account; therefore, the RBDO leads to economical and safe design. It offers a good alternative to the safety factor approach, which is based on deterministic considerations and cannot take account for reduction of safety margins during the optimization procedure. In RBDO models, there are two kinds of variables:

- The design variables $d_{k}$ which are the deterministic variables to be defined in order to optimize the design. They represent the control parameters of the mechanical system (e.g. dimensions, materials, loads) and of the probabilistic model (e.g. means and standard deviations of the random variables).

- The random variables $x_{i}$ which represent the structural uncertainties, identified by probabilistic distributions. These variables can be related to geometrical dimensions, material characteristics and applied external loading.

Basically, the RBDO aims at minimizing the total expected cost $C_{T}$ (see Figure 9) which is given in terms of initial manufacturing and construction costs $C_{c}$ and direct failure cost $C_{f}$ :

$$
C_{T}=C_{c} \boldsymbol{\bigotimes}_{k}{ }^{-}+C_{f} P_{f} \boldsymbol{\varangle}_{i}, d_{k}^{-}
$$


B. Yannou, N. Troussier, A. Chateauneuf, N. Boudaoud, D. Scaravetti

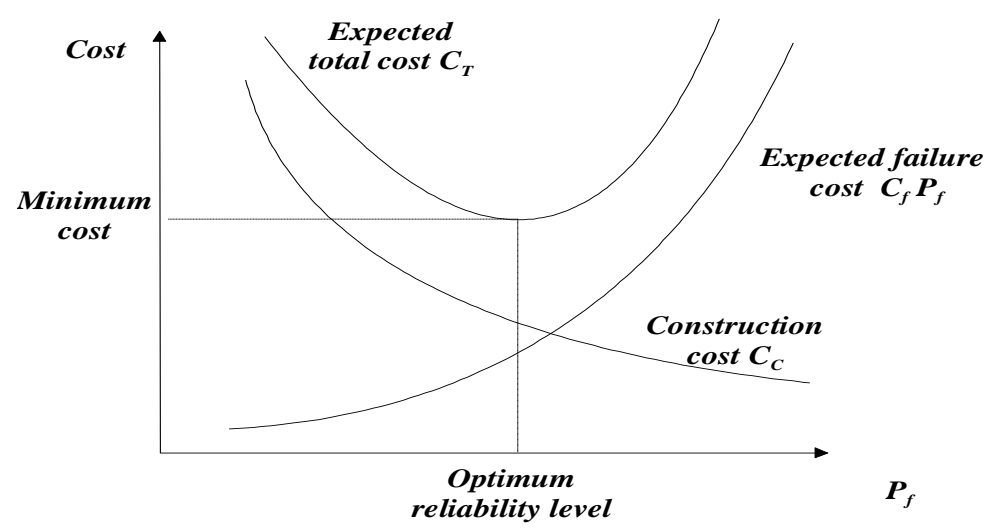

Figure 9 Expected total cost in terms of failure probability

Due to difficulties in the failure cost estimation $C_{f}$ (especially when dealing with human lives), the direct use of the above equation is not that easy. A practical formulation consists in minimizing the initial cost under the constraint of satisfying a target safety level $\beta_{t}$ :

$$
\left\{\begin{array}{l}
\min _{d_{k}} C_{c} \mathbb{Q}_{k}- \\
\text { subject to: } \quad \beta \mathbb{Q}_{k}, x_{i} \geq \beta_{t} \\
\\
d_{k}^{L} \leq d_{k} \leq d_{k}^{U}
\end{array}\right.
$$

where $d_{k}{ }^{L}$ and $d_{k}{ }^{U}$ are respectively the lower and upper bounds of the $k^{\text {th }}$ design variable.

This formulation represents two embedded optimization problems. The outer one concerns the search for optimal design variables to minimize the cost and the inner one concerns the evaluation of the reliability index in the space of random variables. The coupling between the optimization and reliability problems is a complex task and leads to a very high calculation cost. The major difficulty lies in the evaluation of the structural reliability, which is carried out by a particular optimization procedure. In the random variable space, the reliability analysis implies a large number of mechanical calls, where in the design variable space, the search procedure modifies the structural configuration and hence requires the re-evaluation of the reliability level at each iteration. For this reason, the solution of these two problems (optimization and reliability) requires very important computation resources that seriously reduces the applicability of this approach. 
Dimensioning a product in preliminary design

\subsection{Application to truss structure}
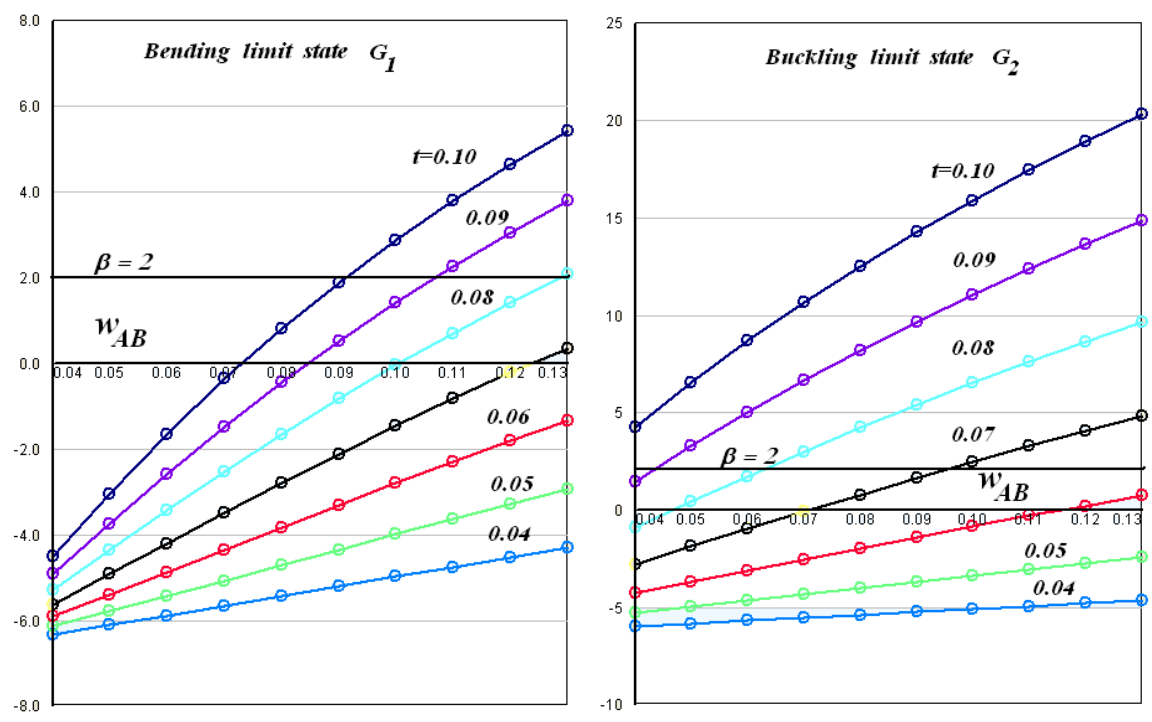

Figure 10 Reliability index evolution in terms of truss dimensions

The truss structure illustrated in figure 1 is now optimized by considering uncertainties. The structural limit states are written:

$$
\begin{aligned}
& G_{1}=f_{Y}-\sigma_{b}\left(w_{A B}, L, W\right) \\
& G_{2}=F_{b} \lessdot w_{A B}, L-F_{A B} \ll w_{A B}, L, W^{-}
\end{aligned}
$$

where $f_{Y}$ is the yield stress, $F_{b}$ is the buckling load of member $A B, \sigma_{b}$ is the bending stress at point $B$ and $F_{A B}$ is the normal force in member $A B$ (detailed expressions are given in section 2 of the paper). For a target reliability $\beta_{t}=2$ (corresponding to a failure probability of $1 \%$ ), the reliability-based optimization problem is written:

$$
\left\{\begin{array}{l}
\min _{t, w_{A B}} M=W_{A B}+W_{C D}=\rho g t L\left(\frac{4 \sqrt{3}}{9} w_{A B}+w_{A B}-0.025\right) \\
\text { subject to }: \beta_{1} \geq 2 \quad \text { and } \quad \beta_{2} \geq 2
\end{array}\right.
$$

where $\beta_{1}$ and $\beta_{2}$ are the reliability indexes related to $G_{1}$ and $G_{2}$, respectively. In this example, the uncertainties are related to the applied load $W$, the material strength $f_{Y}$ and the truss length $L$, where the coefficients of variation are respectively $15 \%, 8 \%$ and $2 \%$.

Figure 10 shows the reliability index evolution for the two limit states, in terms of the design variables $w_{A B}$ and $t$. For the required safety level $\beta \geq 2$, the safe design space is reduced to points above this level. The search for the minimum weight in this subspace leads to the optimal solution given by: $w_{A B}=0.091 \mathrm{~m}$ and $t=0.10 \mathrm{~m}$, corresponding to a global mass of $4180 \mathrm{~kg}$. At this solution, the bending limit state is observed as the most critical one. This result also indicates a safety factor of 1.38 for this limit state. 


\section{B. Yannou, N. Troussier, A. Chateauneuf, N. Boudaoud, D. Scaravetti}

\section{Concluding remarks: Three complementary approaches for embodiment design}

The studied approaches of parametric design exploration have shown to be complementary and interactive. The exploration of the design space allows us to define the region of potentially interesting feasible solutions, which can be efficiently used for further investigations. The use of metamodels becomes thus precise as the search region is shrunk. These metamodels can then be used for multi-disciplinary design considering several performance objectives and constraints. As the design solution is almost described, it becomes necessary to carry out a design for reliability in order to take parameter and functioning fluctuations into account as well as uncertainties. Finally, the whole process allows us to reach a cost-based robust and reliable design. The application to a simple truss illustrates the advantages and difficulties in the different stages of the proposed process. The three considered successive design stages of design exploration, robust design and design for reliability are more and more sophisticated since they need more and more modeling information to provide a result. This is the reason why they must be successively used within a design process. Indeed, applying one of the three approaches allows to quickly figure out inadequacies with performance specifications or initial allowable bounds of design parameters and then to backtrack or to refine the design issue before passing to the next stage or approach. First, the design space is more and more precisely defined like in a Toyota-like set-based approach of design under uncertainty - see (Ward et al. 1994; Finch et al. 1997) -. Second, we clearly show that the successive optimal designs obtained by the three categories of methods are notably different, but that the optimal point obtained in a given approach is used to explore its surroundings in the next approach. This paper is just an illustration of this progressive and ever complexified preliminary parametric design process.

Table 4 summarizes that the best considered designs are significantly different in the three design stages. The comparison of the different methods is not that easy, as each one is based on specific assumptions. For example, the reliable design indicates that the safety factor should be reduced to 1.38 instead of 1.506; this leads to enlarge the design exploration space. However, if the designer requires higher reliability levels, the safety factor is automatically reduced at the optimal design.

Table 4 The best solutions found during the stages of design exploration, robust and reliable design

\begin{tabular}{|l|l|l|l|l|l|l|l|l|}
\hline & $t$ & $w_{A B}$ & $L$ & $W$ & $h$ & $\alpha$ & $M$ & $s$ \\
\hline $\begin{array}{l}\text { Opportunistic } \\
\text { Design } \\
\text { exploration } \\
\text { (given in } \\
\text { (Yannou and } \\
\text { Hamdi, 2004)) }\end{array}$ & 0.0995 & 0.0927 & 3.01 & 16570 & & 3190 & 1.506 \\
\hline Robust design & 0.10 & 0.13 & 3.42 & 17115 & 1.5 & 0.5 & & \\
\hline Reliable design & 0.10 & 0.091 & 4.00 & 15385 & & & 4180 & 1.38 \\
\hline
\end{tabular}




\section{Dimensioning a product in preliminary design}

\section{References}

Antonsson, E. K. and Otto, K. N. (1995) 'Imprecision in Engineering Design', Journal of Mechanical Design, Vol. 117, No. B, pp.25-32.

Aoues, Y. and Chateauneuf, A. (2007, in Press) Reliability-based optimization of structural systems by adaptive target safety - application to RC frames, Structural Safety.

Benhamou, F., Goualard, F., Granvilliers, L. and Puget, J.-F. (1999) 'Revising Hull and Box Consistency' In Procs. of ICLP'99The MIT Press, Las Cruces, USA.

Crossland, R., Sims, W. J. H. and McMahon, C. A. (2003) 'An object-oriented modeling framework for representing uncertainty in early variant design', Research in Engineering Design, Vol. 14, pp.173-183.

El-Haik, B. (2005) Axiomatic Quality: Integrating Axiomatic Design with Six-Sigma, Reliability, and Quality Engineering, Wiley-Interscience, ISBN-10: 047168273X.

Finch, W. W. and Ward, A. C. (1997) 'A set-based system for eliminating infeasible designs in engineering problems dominated by uncertainty', Proceedings of the DETC'97: ASME / Design Engineering Technical Conference, Sacramento, California, pp.DETC97/DTM-3886.

Flowkes, W. Y. and Creveling, C. M. (1995) Engineering methods for robust product design: using Taguchi methods in technology and product development, Addison-Wesley Pub. Co., ISBN 0201633671.

Fowlkes, W. Y. and Creveling, C. M. (1995) Engineering Methods for Robust Product Design: Using Taguchi Methods in Technology and Product Development, Addison-Wesley publisher, Hardcover format, ISBN: 0201633671.

Kharmanda, G., Chateauneuf, A. and Lemaire, M. (2002) 'Efficient reliability-based design optimization using a hybrid space with application to finite element analysis', 24, pp.233245.

Messac, A., Ismail-Yahaya, A. and Mattson, C. A. (2003) 'The Normalized Normal Constraint Method for Generating the Pareto Frontier', Structural and Multidisciplinary Optimization Journal of the International Society of Structural and Multidisciplinary Optimization (ISSMO), Vol. 25, No. 2, pp.86-98.

Moore, R. E. (1979) Methods and Applications of Interval Analysis, SIAM, Philadelphia.

Pahl, B. and Beitz, W. (1996) Engineering Design: A Systematic Approach, Springer, Technology \& Industrial Arts, ISBN 3540199179.

Papalambros, P. Y. (2002) 'The optimization paradigm in engineering design: promises and challenges', Computer-Aided Design, Vol. 34, No. 12, pp.939-951.

Scaravetti, D., Pailhes, P. and Nadeau, J.-P. (2006a) 'Expression de la pertinence de la conception, pour l'aide à la décision en conception préliminaire' In Evaluation et décision dans le processus de conception (chapitre 3)(Eds, Yannou, B. and Bonjour, E.) Hermès, ISBN: 27462-1394-X, pp.53-68.

Scaravetti, D., Sébastian, P., Pailhes, J. and Nadeau, J.-P. (2006b) 'Exploring design spaces in the search for embodiment design solutions for various life cycle stages, and decision support', Proceedings of the Computational Engineering in Systems Applications (CESA'06), Beijing, China, October 2006.

Scott, M. J. and Antonsson, E. K. (2000) 'Using Indifference Points in Engineering Decisions', Proceedings of the ASME/DETC2000/DTM, Baltimore, Maryland, USA, September 10-13.

Stump, G., Yukish, M. A., Martin, J. D. and Simpson, T. W. (2004) 'The ARL Trade Space Visualizer - An Engineering Decision-Making Tool', Proceedings of the 10th AIAA/ISSMO Multidisciplinary Analysis and Optimization Conference, Albany, NY, Aug. 30 - Sep. 1.

Suh, N. (2001) Axiomatic Design: Advances and Applications, Oxford University Press, ISBN-10: 0195134664, New York.

Ullman, D. G. and D'ambrosio, B. (1995) 'Taxonomy for Classifying Engineering Decision Problems and Support Systems', Artificial Intelligence for Engineering Design, Analysis and Manufacturing, Vol. 9, pp.427-438. 


\section{B. Yannou, N. Troussier, A. Chateauneuf, N. Boudaoud, D. Scaravetti}

Ward, A. C., Liker, J. K., Sobek, D. K. and Cristiano, J. J. (1994) 'Set-based concurrent engineering and Toyota', Proceedings of the DETC'94: ASME / Design Engineering Technical Conference, Sacramento, California, pp.DETC94/DTM, 79-90.

Wood, K. L. and Antonsson, E. K. (1989) 'Computations with imprecise parameters in engineering design: background and theory', ASME Journal of Mechanisms, Transmissions and Automation in Design, Vol. 111, No. 4, pp.616-625.

Yannou, B. and Hamdi, A. (2004) 'Truss dimensioning with an uncertainty reduction paradigm', Proceedings of the International Design Conference, Dubrovnik, Croatia, May 18-20.

Yannou, B. and Harmel, G. (2005) 'Use of Constraint Programming for Design' In Advances in Design (Eds, ElMaraghy, H. and ElMaraghy, W.) Springer, Chapter 12.

Yannou, B., Moreno, F., Thévenot, H. and Simpson, T. W. (2005) 'Faster Generation of Feasible Design Points', Proceedings of the DETC/DAC: ASME Design Engineering Technical Conferences / Design Automation Conference, Long Beach, CA, USA, Sept. 25-28, pp.DETC2005/85449. 